\title{
ON CONVERGENCE OF CHLODOWSKY-TYPE MKZD OPERATORS
}

\author{
HARUN KARSLI
}

\begin{abstract}
In the present paper, we define a new kind of MKZD operators for functions defined on $\left[0, b_{n}\right]$, named Chlodowsky type MKZD operators and give an estimate, by means of the techniques of probability theory, on the rate of convergence of operators $M_{n}^{*}$ for functions of bounded variation on the interval $\left[0, b_{n}\right],(n \rightarrow \infty)$ extending infinity.
\end{abstract}

\section{Introduction}

For a function defined on the interval $[0,1]$, the Meyer-König and Zeller operators $M_{n}(f, x)[1]$ are defined as

$$
M_{n}(f ; x)=\sum_{k=0}^{\infty} m_{n, k}(x) f\left(\frac{k}{n+k}\right)
$$

where $m_{n, k}(x)=\left(\begin{array}{c}n+k-1 \\ k\end{array}\right) x^{k}(1-x)^{n}$. In 1989 Guo [2] introduced the integrated Meyer-König and Zeller operators $\widetilde{M}_{n}$ by the means of the operators (1.1), to approximate Lebesgue integrable functions on the interval $[0,1]$, are defined as

$$
\tilde{M}_{n}(f ; x)=\sum_{k=0}^{\infty} \tilde{m}_{n, k}(x) \int_{I_{k}} f(t) d t
$$

where $I_{k}=\left[\frac{k}{n+k}, \frac{k+1}{n+k+1}\right]$ and $\tilde{m}_{n, k}(x)=(n+1)\left(\begin{array}{c}n+k+1 \\ k\end{array}\right) x^{k}(1-x)^{n}$. The rates of pointwise convergence of operators (1.2) and some other integral modifications of the MKZ operators defined by (1.1) has been studied for functions of bounded variation on $[0,1]$. We refer to the reader [3]-[8]. We also mention some of the important papers on similar type of operators are due to Zeng and Cheng [10],

Received by the editors April 09, 2007; Rev: Feb. 13, 2008 Accepted: March 20, 2008.

2000 Mathematics Subject Classification. Primary 41A25.; Secondary 41A36.

Key words and phrases. Rate of convergence, Approximation, MKZD operators, Chlodowskytype MKZD operators, Bounded variation. 
Bojanic and Vuilleumier [11], Cheng [12], Gupta and Gupta et al. [13,14]. It is useful to mention very recent papers by Karsli and Ibikli [15-16], which were dealt with the rate of pointwise convergence of the Chlodowsky-type Bernstein operators and its Bézier variant in the space $B V\left[0, b_{n}\right]$ respectively.

Recently, Ibikli and Karsli [17] have defined Chlodowsky type Durrmeyer operators $D_{n}$, for a function defined on the interval $\left[0, b_{n}\right]$, as: $D_{n}: B V[0, \infty) \rightarrow \mathcal{P}$,

$$
D_{n}(f ; x)=\frac{(n+1)}{b_{n}} \sum_{k=0}^{n} P_{n, k}\left(\frac{x}{b_{n}}\right) \int_{0}^{b_{n}} f(t) P_{n, k}\left(\frac{t}{b_{n}}\right) d t, 0 \leq x \leq b_{n}
$$

where $\mathcal{P}:=\{P:[0, \infty) \rightarrow R\}$, is a polynomial function, $\left(b_{n}\right)$ is a positive increasing sequence with the properties

$$
\lim _{n \rightarrow \infty} b_{n}=\infty \text { and } \lim _{n \rightarrow \infty} \frac{b_{n}}{n}=0
$$

and $P_{n, k}(x)=\left(\begin{array}{c}n \\ k\end{array}\right) x^{k}(1-x)^{n-k}$ is the Bernstein basis. They also estimated in the same paper, the rate of convergence of the operators (1.3) for functions in $B V[0, \infty)$.

In the present paper, we define a new kind of MKZD operators for functions defined on $\left[0, b_{n}\right]$, named Chlodowsky-type MKZD operators as

$$
M_{n}^{*}(f ; x)=\sum_{k=0}^{\infty} \frac{n+k}{b_{n}} m_{n, k}\left(\frac{x}{b_{n}}\right) \int_{0}^{b_{n}} f(t) b_{n, k}\left(\frac{t}{b_{n}}\right) d t, 0 \leq x \leq b_{n}
$$

where $\left(b_{n}\right)$ is a positive increasing sequence with the properties

$$
\lim _{n \rightarrow \infty} b_{n}=\infty \text { and } \lim _{n \rightarrow \infty} \frac{b_{n}}{n}=0
$$

and $b_{n, k}(t)=n\left(\begin{array}{c}n+k \\ k\end{array}\right) t^{k}(1-t)^{n-1}$.

The aim of this paper is to study the behavior of the $M_{n}^{*}$ operators defined by (1.4) for functions of bounded variation and give an estimate, by means of the techniques of probability theory, on the rate of convergence of the operators (1.4) on the interval $\left[0, b_{n}\right],(n \rightarrow \infty)$ extending infinity.

For the sake of brevity, let the auxiliary function $f_{x}$ be defined by

$$
f_{x}(t)=\left\{\begin{array}{cl}
f(t)-f(x+) & , \quad x<t \leq b_{n} \\
0 & , \quad t=x \\
f(t)-f(x-) & , 0 \leq t<x
\end{array}\right.
$$

The main theorem of this paper is as follows. 
Theorem. Let $f$ be a function of bounded variation on every finite subinterval of $[0, \infty)$. Then for every $x \in(0, \infty)$, and $n$ sufficiently large, we have,

$$
\begin{aligned}
\left|M_{n}^{*}(f ; x)-\frac{1}{2}(f(x+)+f(x-))\right| \leq & \frac{3 A_{n}(x) b_{n}^{2}}{x^{2}\left(b_{n}-x\right)^{2}}\left(\sum_{k=1}^{n} \bigvee_{x-\frac{x}{\sqrt{k}}}^{x+\frac{b_{n}-x}{\sqrt{k}}}\left(f_{x}\right)\right) \\
& +|f(x+)-f(x-)| \frac{1}{\sqrt{2 e}} \frac{1}{\sqrt{n \frac{x}{b_{n}}}}
\end{aligned}
$$

where

$$
A_{n}(x)=\frac{4 x b_{n}}{n-1}+\frac{2 b_{n}^{2}}{(n-1)(n-2)}+\frac{4 x^{2}}{n+1}
$$

and $\bigvee_{a}^{b}\left(f_{x}\right)$ is the total variation of $f_{x}$ on $[a, b]$

\section{Auxiliary Results}

In this section we give certain results, which are necessary to prove our main theorem.

Lemma 1. For $s \in \mathbb{N}$

$$
M_{n}^{*}\left(t^{s} ; x\right)=b_{n}^{s} \sum_{k=0}^{\infty} m_{n, k}\left(\frac{x}{b_{n}}\right) \frac{(n+k) !}{k !} \frac{(k+s) !}{(k+s+n) !} .
$$

Proof. We have

$$
\begin{aligned}
M_{n}^{*}\left(t^{s} ; x\right) & =\sum_{k=0}^{\infty} \frac{n+k}{b_{n}} m_{n, k}\left(\frac{x}{b_{n}}\right)\left[\int_{0}^{b_{n}} b_{n, k}\left(\frac{t}{b_{n}}\right) t^{s} d t\right] \\
& =\sum_{k=0}^{\infty} \frac{n+k}{b_{n}} m_{n, k}\left(\frac{x}{b_{n}}\right)\left[\int_{0}^{b_{n}}\left(\begin{array}{c}
n+k-1 \\
k
\end{array}\right)\left(\frac{t}{b_{n}}\right)^{k}\left(1-\frac{t}{b_{n}}\right)^{n-1} t^{s} d t\right]
\end{aligned}
$$


Setting $u=\frac{t}{b_{n}}$, we see that

$$
\begin{aligned}
M_{n}^{*}\left(t^{s} ; x\right) & =\sum_{k=0}^{\infty} \frac{n+k}{b_{n}} m_{n, k}\left(\frac{x}{b_{n}}\right) b_{n}^{s+1}\left(\begin{array}{c}
n+k-1 \\
k
\end{array}\right) \int_{0}^{1} u^{k+s}(1-u)^{n-1} d u \\
& =\sum_{k=0}^{\infty}(n+k) m_{n, k}\left(\frac{x}{b_{n}}\right) b_{n}^{s}\left(\begin{array}{c}
n+k-1 \\
k
\end{array}\right) B(k+s+1, n) \\
& =b_{n}^{s} \sum_{k=0}^{\infty}(n+k) m_{n, k}\left(\frac{x}{b_{n}}\right) \frac{(n+k-1) !}{(n-1) ! k !} \frac{(k+s) !(n-1) !}{(k+s+n) !} \\
& =b_{n}^{s} \sum_{k=0}^{\infty} m_{n, k}\left(\frac{x}{b_{n}}\right) \frac{(n+k) !}{k !} \frac{(k+s) !}{(k+s+n) !} .
\end{aligned}
$$

For $s=0,1$ and 2 in (2.1), we get respectively

$$
\begin{aligned}
M_{n}^{*}(1 ; x)=\sum_{k=0}^{\infty} m_{n, k}\left(\frac{x}{b_{n}}\right)=1, \\
M_{n}^{*}(t ; x)=b_{n} \sum_{k=0}^{\infty} m_{n, k}\left(\frac{x}{b_{n}}\right) \frac{(n+k) !}{k !} \frac{(k+1) !}{(k+1+n) !} \\
=\left(1-\frac{x}{b_{n}}\right)^{n} b_{n} \sum_{k=0}^{\infty} \frac{(n+k-1) !}{(n-1) ! k !}\left(\frac{x}{b_{n}}\right)^{k} \frac{k+1}{k+1+n} \\
=\left(1-\frac{x}{b_{n}}\right)^{n} b_{n} \sum_{k=1}^{\infty} \frac{(n+k-2) !}{(n-1) !(k-1) !}\left(\frac{x}{b_{n}}\right)^{k} \frac{k+1}{k+1+n} \frac{n+k-1}{k} \\
=\left(1-\frac{x}{b_{n}}\right)^{n} b_{n} \sum_{k=1}^{\infty} \frac{(n+k-2) !}{(n-1) !(k-1) !}\left(\frac{x}{b_{n}}\right)^{k} \frac{k+1}{k+1+n}\left[1-\frac{2}{n+k+1}\right] \\
=\left(1-\frac{x}{b_{n}}\right)^{n} b_{n} \sum_{k=1}^{\infty} \frac{(n+k-2) !}{(n-1) !(k-1) !}\left(\frac{x}{b_{n}}\right)^{k} \frac{k+1}{k+1+n}\left[1-\frac{2}{n+k+1}\right] \\
\geq\left(1-\frac{x}{b_{n}}\right)^{n} b_{n} \sum_{k=1}^{\infty} \frac{(n+k-2) !}{(n-1) !(k-1) !}\left(\frac{x}{b_{n}}\right)^{k} \frac{k+1}{k+1+n}\left[1-\frac{2}{n+1}\right] \\
\geq\left[1-\frac{2}{n+1}\right]\left(1-\frac{x}{b_{n}}\right)^{n} b_{n} \sum_{k=1}^{\infty} \frac{(n+k-2) !}{(n-1) !(k-1) !}\left(\frac{x}{b_{n}}\right)^{k}
\end{aligned}
$$




$$
\begin{aligned}
& =\left[1-\frac{2}{n+1}\right]\left(1-\frac{x}{b_{n}}\right)^{n} b_{n} \sum_{k=0}^{\infty} \frac{(n+k-1) !}{(n-1) ! k !}\left(\frac{x}{b_{n}}\right)^{k+1} \\
& =\left[1-\frac{2}{n+1}\right] \frac{x}{b_{n}} b_{n} \sum_{k=0}^{\infty} \frac{(n+k-1) !}{(n-1) ! k !}\left(\frac{x}{b_{n}}\right)^{k}\left(1-\frac{x}{b_{n}}\right)^{n} \\
& =\left[1-\frac{2}{n+1}\right] x \sum_{k=0}^{\infty}\left(\begin{array}{c}
n+k-1 \\
k
\end{array}\right)\left(\frac{x}{b_{n}}\right)^{k}\left(1-\frac{x}{b_{n}}\right)^{n} \\
& =\left[1-\frac{2}{n+1}\right] x
\end{aligned}
$$

and

$$
\begin{aligned}
& M_{n}^{*}\left(t^{2} ; x\right)=b_{n}^{2} \sum_{k=0}^{\infty} m_{n, k}\left(\frac{x}{b_{n}}\right) \frac{(n+k) !}{k !} \frac{(k+2) !}{(k+2+n) !} \\
& =\left(1-\frac{x}{b_{n}}\right)^{n} b_{n}^{2} \sum_{k=0}^{\infty} \frac{(n+k-1) !}{(n-1) ! k !}\left(\frac{x}{b_{n}}\right)^{k} \frac{(n+k) !}{k !} \frac{(k+2) !}{(k+2+n) !} \\
& =\left(1-\frac{x}{b_{n}}\right)^{n} b_{n}^{2} \sum_{k=0}^{\infty} \frac{(n+k-1) !}{(n-1) ! k !}\left(\frac{x}{b_{n}}\right)^{k} \frac{(k+2)(k+1)}{(k+2+n)(k+1+n)} \\
& =\left(1-\frac{x}{b_{n}}\right)^{n} b_{n}^{2} \sum_{k=0}^{\infty} \frac{(n+k-1) !}{(n-1) ! k !}\left(\frac{x}{b_{n}}\right)^{k} \frac{k(k-1)+4 k+2}{(k+2+n)(k+1+n)} \\
& \leq\left(1-\frac{x}{b_{n}}\right)^{n} b_{n}^{2} \frac{1}{(n-1) !} \sum_{k=2}^{\infty} \frac{(n+k-3) !}{(k-2) !}\left(\frac{x}{b_{n}}\right)^{k} \\
& +4\left(1-\frac{x}{b_{n}}\right)^{n} b_{n}^{2} \sum_{k=1}^{\infty} \frac{(n+k-3) !}{(k-1) !}\left(\frac{x}{b_{n}}\right)^{k} \\
& +2\left(1-\frac{x}{b_{n}}\right)^{n} b_{n}^{2} \sum_{k=0}^{\infty} \frac{(n+k-3) !}{k !}\left(\frac{x}{b_{n}}\right)^{k} \\
& \leq\left(1-\frac{x}{b_{n}}\right)^{n} b_{n}^{2}\left[\sum_{k=0}^{\infty} \frac{(n+k-1) !}{(n-1) ! k !}\left(\frac{x}{b_{n}}\right)^{k+2}+\frac{4}{n-1} \sum_{k=0}^{\infty} \frac{(n+k-1) !}{(n-1) ! k !}\left(\frac{x}{b_{n}}\right)^{k+1}\right. \\
& \left.+\frac{2}{(n-1)(n-2)} \sum_{k=0}^{\infty} \frac{(n+k-1) !}{(n-1) ! k !}\left(\frac{x}{b_{n}}\right)^{k}\right]
\end{aligned}
$$




$$
\begin{aligned}
= & x^{2} \sum_{k=0}^{\infty} \frac{(n+k-1) !}{(n-1) ! k !}\left(\frac{x}{b_{n}}\right)^{k}\left(1-\frac{x}{b_{n}}\right)^{n}+\frac{4 x b_{n}}{n-1} \sum_{k=0}^{\infty} \frac{(n+k-1) !}{(n-1) ! k !}\left(\frac{x}{b_{n}}\right)^{k}\left(1-\frac{x}{b_{n}}\right)^{n} \\
& +\frac{2 b_{n}^{2}}{(n-1)(n-2)} \sum_{k=0}^{\infty} \frac{(n+k-1) !}{(n-1) ! k !}\left(\frac{x}{b_{n}}\right)^{k}\left(1-\frac{x}{b_{n}}\right)^{n} \\
= & x^{2}+\frac{4 x b_{n}}{n-1}+\frac{2 b_{n}^{2}}{(n-1)(n-2)} .
\end{aligned}
$$

From (2.2), (??) and (2.3), an easy computation gives

$$
\left|M_{n}^{*}((t-x) ; x)\right|=\frac{2 x}{n+1}
$$

and

$$
\begin{aligned}
M_{n}^{*}\left((t-x)^{2} ; x\right) & \leq x^{2}+\frac{4 x b_{n}}{n-1}+\frac{2 b_{n}^{2}}{(n-1)(n-2)}-2\left[1-\frac{2}{n+1}\right] x^{2}+x^{2} \\
& =\frac{4 x b_{n}}{n-1}+\frac{2 b_{n}^{2}}{(n-1)(n-2)}+\frac{4 x^{2}}{n+1}:=A_{n}(x) .
\end{aligned}
$$

Recently Zeng [9] estimated the exact bounds for Bernstein basis functions and Meyer Konig Zeller basis functions.For $k \in \mathbb{N}$ and $t \in(0,1]$, the author [9] obtained the inequality:

$$
\left(\begin{array}{c}
n+k-1 \\
k
\end{array}\right) t^{k}(1-t)^{n}<\frac{1}{\sqrt{2 e}} \frac{1}{\sqrt{n t}} .
$$

Replacing the variable $t$ with $\frac{t}{b_{n}}$, in eq.(2.5) we get the following result:

Lemma 2. For all $t \in\left(0, b_{n}\right]$ and $k \in \mathbb{N}$, we have

$$
\left(\begin{array}{c}
n+k-1 \\
k
\end{array}\right)\left(\frac{t}{b_{n}}\right)^{k}\left(1-\frac{t}{b_{n}}\right)^{n}<\frac{1}{\sqrt{2 e}} \frac{1}{\sqrt{n \frac{t}{b_{n}}}}
$$

In the following statements (and throughout the paper), we use the notations;

$$
K_{n}\left(\frac{x}{b_{n}}, \frac{u}{b_{n}}\right):=\sum_{k=0}^{\infty} \frac{n+k}{b_{n}} m_{n, k}\left(\frac{x}{b_{n}}\right) b_{n, k}\left(\frac{u}{b_{n}}\right)
$$

and

$$
\lambda_{n}\left(\frac{x}{b_{n}}, \frac{t}{b_{n}}\right):=\int_{0}^{t} K_{n}\left(\frac{x}{b_{n}}, \frac{u}{b_{n}}\right) d u .
$$

Here we note that

$$
\lambda_{n}\left(\frac{x}{b_{n}}, 1\right)=\int_{0}^{b_{n}} K_{n}\left(\frac{x}{b_{n}}, \frac{u}{b_{n}}\right) d u=1 .
$$


Lemma 3. For all $x \in\left(0, b_{n}\right)$, we have

$$
\begin{aligned}
\lambda_{n}\left(\frac{x}{b_{n}}, \frac{y}{b_{n}}\right) & =\int_{0}^{y} K_{n}\left(\frac{x}{b_{n}}, \frac{u}{b_{n}}\right) d u \leq \frac{A_{n}(x)}{(x-y)^{2}}, \quad 0 \leq y<x, \\
1-\lambda_{n}\left(\frac{x}{b_{n}}, \frac{z}{b_{n}}\right) & =\int_{z}^{1} K_{n}\left(\frac{x}{b_{n}}, \frac{u}{b_{n}}\right) d u \leq \frac{A_{n}(x)}{(z-x)^{2}}, \quad x<z \leq 1
\end{aligned}
$$

where $A_{n}(x)$ is defined in Theorem.

Proof. We first prove (2.8) as follows:

$$
\begin{aligned}
\lambda_{n}\left(\frac{x}{b_{n}}, \frac{y}{b_{n}}\right) & =\int_{0}^{y} K_{n}\left(\frac{x}{b_{n}}, \frac{u}{b_{n}}\right) d u \leq \int_{0}^{y} K_{n}\left(\frac{x}{b_{n}}, \frac{u}{b_{n}}\right)\left(\frac{x-u}{x-y}\right)^{2} d u \\
& =\frac{1}{(x-y)^{2}} M_{n}^{*}\left((u-x)^{2} ; x\right) .
\end{aligned}
$$

By (2.4), we have

$$
\lambda_{n}\left(\frac{x}{b_{n}}, \frac{y}{b_{n}}\right) \leq \frac{A_{n}(x)}{(x-y)^{2}} .
$$

The proof of (2.9) is analogous.

\section{Main Result}

Proof. For any $f(t) \in\left[0, b_{n}\right]$, from (1.5) it is clear that

$$
\begin{aligned}
f(t)= & \frac{1}{2}(f(x+)+f(x-))+f_{x}(t)+\frac{f(x+)-f(x-)}{2} \operatorname{sgn}(t-x) \\
& +\delta_{x}(t)\left[f(x)-\frac{1}{2}(f(x+)+f(x-))\right] .
\end{aligned}
$$

Applying the operator (1.4) to (3.1), we have

$$
\begin{gathered}
M_{n}^{*}(f ; x)=\frac{1}{2}(f(x+)+f(x-)) M_{n}^{*}(1 ; x)+M_{n}^{*}\left(f_{x} ; x\right) \\
+\frac{f(x+)-f(x-)}{2} M_{n}^{*}(\operatorname{sgn}(t-x) ; x)+\left[f(x)-\frac{1}{2}(f(x+)+f(x-))\right] M_{n}^{*}\left(\delta_{x} ; x\right) .
\end{gathered}
$$

Hence

$$
\begin{gathered}
\left|M_{n}^{*}(f ; x)-\frac{1}{2}(f(x+)+f(x-)) M_{n}^{*}(1 ; x)\right| \leq\left|M_{n}^{*}\left(f_{x} ; x\right)\right| \\
+\left|\frac{f(x+)-f(x-)}{2}\right|\left|M_{n}^{*}(\operatorname{sgn}(t-x) ; x)\right|+\left|f(x)-\frac{1}{2}(f(x+)+f(x-))\right|\left|M_{n}^{*}\left(\delta_{x} ; x\right)\right| .
\end{gathered}
$$


For operators $M_{n}^{*}(f ; x)$, it is obvious that $M_{n}^{*}\left(\delta_{x} ; x\right)=0$. From this fact, we can write

$\left|M_{n}^{*}(f ; x)-\frac{1}{2}(f(x+)+f(x-))\right| \leq\left|M_{n}^{*}\left(f_{x} ; x\right)\right|+\left|\frac{f(x+)-f(x-)}{2}\right|\left|M_{n}^{*}(\operatorname{sgn}(t-x) ; x)\right|$.

In order to prove the main theorem, we need the estimates for $M_{n}^{*}\left(f_{x} ; x\right)$ and $M_{n}^{*}(\operatorname{sgn}(t-x) ; x)$ in $(3.2)$.

Firstly, we evaluate $M_{n}^{*}\left(f_{x} ; x\right)$ as follows:

$$
\begin{aligned}
\left|M_{n}^{*}\left(f_{x} ; x\right)\right|= & \left|\sum_{k=0}^{\infty} \frac{n+k}{b_{n}} m_{n, k}\left(\frac{x}{b_{n}}\right)\left[\int_{0}^{x-\frac{x}{\sqrt{n}}}+\int_{x-\frac{x}{\sqrt{k}}}^{x+\frac{b_{n}-x}{\sqrt{n}}}+\int_{x+\frac{b_{n}-x}{\sqrt{n}}}^{b_{n}} b_{n, k}\left(\frac{t}{b_{n}}\right) f_{x}(t) d t\right]\right| \\
\leq & \left|\sum_{k=0}^{\infty} \frac{n+k}{b_{n}} m_{n, k}\left(\frac{x}{b_{n}}\right) \int_{0}^{x-\frac{x}{\sqrt{n}}} b_{n, k}\left(\frac{t}{b_{n}}\right) f_{x}(t) d t\right| \\
& +\left|\sum_{k=0}^{\infty} \frac{n+k}{b_{n}} m_{n, k}\left(\frac{x}{b_{n}}\right) \int_{x-\frac{x}{\sqrt{k}}}^{x+\frac{b_{n}-x}{\sqrt{n}}} b_{n, k}\left(\frac{t}{b_{n}}\right) f_{x}(t) d t\right| \\
& +\left|\sum_{k=0}^{\infty} \frac{n+k}{b_{n}} m_{n, k}\left(\frac{x}{b_{n}}\right) \int_{x+\frac{b_{n}-x}{\sqrt{n}}}^{b_{n}} b_{n, k}\left(\frac{t}{b_{n}}\right) f_{x}(t) d t\right| \\
= & :\left|I_{1}(n, x)\right|+\left|I_{2}(n, x)\right|+\left|I_{3}(n, x)\right| .
\end{aligned}
$$

We shall evaluate $I_{1}(n, x), I_{2}(n, x)$ and $I_{3}(n, x)$.

First we estimate $I_{2}(n, x)$. For $t \in\left[x-\frac{x}{\sqrt{n}}, x+\frac{b_{n}-x}{\sqrt{n}}\right]$, we have from $(2.7)$

$$
\begin{aligned}
\left|I_{2}(n, x)\right| & \leq \int_{x-\frac{x}{\sqrt{k}}}^{x+\frac{b_{n}-x}{\sqrt{n}}}\left|f_{x}(t)-f_{x}(x)\right|\left|d_{t}\left(\lambda_{n}\left(\frac{x}{b_{n}}, \frac{t}{b_{n}}\right)\right)\right| \leq \bigvee_{x-\frac{x}{\sqrt{n}}}^{x+\frac{b_{n}-x}{\sqrt{n}}}\left(f_{x}\right) \\
& \leq \frac{1}{n-1} \sum_{k=2}^{n} \bigvee_{x-\frac{x}{\sqrt{n}}}^{x+\frac{b_{n}-x}{\sqrt{n}}}\left(f_{x}\right)
\end{aligned}
$$


Next, we estimate $I_{1}(n, x)$. Using partial Lebesque-Stieltjes integration, we have again from (2.7)

$I_{1}(n, x)=f_{x}\left(x-\frac{x}{\sqrt{n}}\right) \lambda_{n}\left(\frac{x}{b_{n}}, \frac{x-\frac{x}{\sqrt{n}}}{b_{n}}\right)-f_{x}(0) \lambda_{n}\left(\frac{x}{b_{n}}, \frac{0}{b_{n}}\right)-\int_{0}^{x-\frac{x}{\sqrt{n}}} \lambda_{n}\left(\frac{x}{b_{n}}, \frac{t}{b_{n}}\right) d_{t}\left(f_{x}(t)\right)$.

Since $\left|f_{x}\left(x-\frac{x}{\sqrt{n}}\right)\right|=\left|f_{x}\left(x-\frac{x}{\sqrt{n}}\right)-f_{x}(x)\right| \leq \bigvee_{x-\frac{x}{\sqrt{n}}}^{x}\left(f_{x}\right)$, it follows that

$$
\left|I_{1}(n, x)\right| \leq \bigvee_{x-\frac{x}{\sqrt{n}}}^{x}\left(f_{x}\right)\left|\lambda_{n}\left(\frac{x}{b_{n}}, \frac{x-\frac{x}{\sqrt{n}}}{b_{n}}\right)\right|+\int_{0}^{x-\frac{x}{\sqrt{n}}} \lambda_{n}\left(\frac{x}{b_{n}}, \frac{t}{b_{n}}\right) d_{t}\left(-\bigvee_{t}^{x}\left(f_{x}\right)\right)
$$

By (2.4), it is clear that

$$
\lambda_{n}\left(\frac{x}{b_{n}}, \frac{x-\frac{x}{\sqrt{n}}}{b_{n}}\right) \leq \frac{1}{\left(\frac{x}{\sqrt{n}}\right)^{2}} A_{n}(x) .
$$

It follows that

$$
\begin{aligned}
\left|I_{1}(n, x)\right| & \leq \bigvee_{x-\frac{x}{\sqrt{n}}}^{x}\left(f_{x}\right) \frac{A_{n}(x)}{\left(\frac{x}{\sqrt{n}}\right)^{2}}+\int_{0}^{x-\frac{x}{\sqrt{n}}} \frac{1}{(x-t)^{2}} A_{n}(x) d_{t}\left(-\bigvee_{t}^{x}\left(f_{x}\right)\right) \\
& =\bigvee_{x-\frac{x}{\sqrt{n}}}^{x}\left(f_{x}\right) \frac{A_{n}(x)}{\left(\frac{x}{\sqrt{n}}\right)^{2}}+A_{n}(x) \int_{0}^{x-\frac{x}{\sqrt{n}}} \frac{1}{(x-t)^{2}} d_{t}\left(-\bigvee_{t}^{x}\left(f_{x}\right)\right)
\end{aligned}
$$

Furthermore, since

$$
\begin{aligned}
\int_{0}^{x-\frac{x}{\sqrt{n}}} \frac{1}{(x-t)^{2}} d_{t}\left(-\bigvee_{t}^{x}\left(f_{x}\right)\right) & =-\left.\frac{1}{(x-t)^{2}} \bigvee_{t}^{x}\left(f_{x}\right)\right|_{0} ^{x-\frac{x}{\sqrt{n}}}+\int_{0}^{x-\frac{x}{\sqrt{n}}} \frac{2}{(x-t)^{3}} \bigvee_{t}^{x}\left(f_{x}\right) d t \\
& =-\frac{1}{\left(\frac{x}{\sqrt{n}}\right)^{2}} \bigvee_{x-\frac{x}{\sqrt{n}}}^{x}\left(f_{x}\right)+\frac{1}{x^{2}} \bigvee_{0}^{x}\left(f_{x}\right)+\int_{0}^{x-\frac{x}{\sqrt{n}}} \frac{2}{(x-t)^{3}} \bigvee_{t}^{x}\left(f_{x}\right) d t
\end{aligned}
$$

Taking $t=x-\frac{x}{\sqrt{u}}$ in the last integral, we get

$$
\int_{0}^{x-\frac{x}{\sqrt{n}}} \frac{2}{(x-t)^{3}} \bigvee_{t}^{x}\left(f_{x}\right) d t=\frac{1}{x^{2}} \int_{1}^{n} \bigvee_{x-\frac{x}{\sqrt{u}}}^{x}\left(f_{x}\right) d u=\frac{1}{x^{2}} \sum_{k=1}^{n} \bigvee_{x-\frac{x}{\sqrt{u}}}^{x}\left(f_{x}\right)
$$


Consequently, we obtain

$$
\left|I_{1}(n, x)\right| \leq \frac{A_{n}(x)}{x^{2}}\left(\bigvee_{0}^{x}\left(f_{x}\right)+\sum_{k=1}^{n} \bigvee_{x-\frac{x}{\sqrt{k}}}^{x}\left(f_{x}\right)\right)
$$

Using a similar method, for evaluating $\left|I_{3}(n, x)\right|$, we find that

$$
\left|I_{3}(n, x)\right| \leq \frac{A_{n}(x)}{\left(b_{n}-x\right)^{2}}\left(\bigvee_{x}^{b_{n}}\left(f_{x}\right)+\sum_{k=1}^{n} \bigvee_{x}^{x+\frac{b_{n}-x}{\sqrt{k}}}\left(f_{x}\right)\right)
$$

Substituting (3.4), (3.5) and (3.6) in (3.3), we obtain

$$
\begin{aligned}
\left|M_{n}^{*}\left(f_{x} ; x\right)\right| & \leq\left|I_{1}(n, x)\right|+\left|I_{2}(n, x)\right|+\left|I_{3}(n, x)\right| \\
& \leq \frac{A_{n}(x) b_{n}^{2}}{x^{2}\left(b_{n}-x\right)^{2}}\left(\bigvee_{0}^{b_{n}}\left(f_{x}\right)+\sum_{k=1}^{n} \bigvee_{x-\frac{x}{\sqrt{k}}}^{x+\frac{b_{n}-x}{\sqrt{k}}}\left(f_{x}\right)\right)+\frac{1}{n-1} \sum_{k=2}^{n} \bigvee_{x-\frac{x}{\sqrt{k}}}^{x+\frac{b_{n}-x}{\sqrt{k}}}\left(f_{x}\right) .
\end{aligned}
$$

On the other hand, note that

$$
\bigvee_{0}^{b_{n}}\left(f_{x}\right) \leq \sum_{k=1}^{n} \bigvee_{x-\frac{x}{\sqrt{k}}}^{x+\frac{b_{n}-x}{\sqrt{k}}}\left(f_{x}\right)
$$

From (3.7), we can rewrite $\left|M_{n}^{*}\left(f_{x} ; x\right)\right|$ as below;

$$
\left|M_{n}^{*}\left(f_{x} ; x\right)\right| \leq \frac{2 A_{n}(x) b_{n}^{2}}{x^{2}\left(b_{n}-x\right)^{2}}\left(\sum_{k=1}^{n} \bigvee_{x-\frac{x}{\sqrt{k}}}^{x+\frac{b_{n}-x}{\sqrt{k}}}\left(f_{x}\right)\right)+\frac{1}{n-1} \sum_{k=2}^{n} \bigvee_{x-\frac{x}{\sqrt{k}}}^{x+\frac{b_{n}-x}{\sqrt{k}}}\left(f_{x}\right)
$$

Note that $\frac{1}{n-1} \leq \frac{A_{n}(x) b_{n}^{2}}{x^{2}\left(b_{n}-x\right)^{2}}$, for $n>1, \frac{x}{b_{n}} \in[0,1]$.

Consequently

$$
\left|M_{n}^{*}\left(f_{x} ; x\right)\right| \leq \frac{3 A_{n}(x) b_{n}^{2}}{x^{2}\left(b_{n}-x\right)^{2}}\left(\sum_{k=1}^{n} \bigvee_{x-\frac{x}{\sqrt{k}}}^{x+\frac{b_{n}-x}{\sqrt{k}}}\left(f_{x}\right)\right)
$$

Now we estimate $M_{n}^{*}(\operatorname{sgn}(t-x) ; x)$. 
By direct calculation, one has

$$
\begin{aligned}
M_{n}^{*}(\operatorname{sgn}(t-x) ; x) & =\sum_{k=0}^{\infty} \frac{n+k}{b_{n}} m_{n, k}\left(\frac{x}{b_{n}}\right)\left[\int_{x}^{b_{n}} b_{n, k}\left(\frac{t}{a_{n}}\right) d t-\int_{0}^{x} b_{n, k}\left(\frac{t}{b_{n}}\right) d t\right] \\
& =\sum_{k=0}^{\infty} \frac{n+k}{b_{n}} m_{n, k}\left(\frac{x}{b_{n}}\right)\left[\int_{0}^{b_{n}} b_{n, k}\left(\frac{t}{b_{n}}\right) d t-2 \int_{0}^{x} b_{n, k}\left(\frac{t}{b_{n}}\right) d t\right] \\
& =1-2 \sum_{k=0}^{\infty} \frac{n+k}{b_{n}} m_{n, k}\left(\frac{x}{b_{n}}\right) \int_{0}^{x} b_{n, k}\left(\frac{t}{b_{n}}\right) d t .
\end{aligned}
$$

Using the fact $\sum_{j=0}^{k} m_{n, j}\left(\frac{x}{b_{n}}\right)=\frac{n+k}{b_{n}} \int_{x}^{b_{n}} b_{n, k}\left(\frac{t}{b_{n}}\right) d t$, one has

$$
M_{n}^{*}(\operatorname{sgn}(t-x) ; x)=1-2 \sum_{k=0}^{\infty} m_{n, k}\left(\frac{x}{b_{n}}\right)\left(1-\sum_{j=0}^{k} m_{n, j}\left(\frac{x}{b_{n}}\right)\right) .
$$

From (2.6), we obtain

$$
\begin{aligned}
\left|M_{n}^{*}(\operatorname{sgn}(t-x) ; x)\right| & =\left|1-2 \sum_{k=0}^{\infty} m_{n, k}\left(\frac{x}{b_{n}}\right)\left[1-\sum_{j=0}^{k} m_{n, j}\left(\frac{x}{b_{n}}\right)\right]\right| \\
& =\left|-1+2 \sum_{k=0}^{\infty} m_{n, k}\left(\frac{x}{b_{n}}\right) \sum_{j=0}^{k} m_{n, j}\left(\frac{x}{b_{n}}\right)\right| \\
& =\left|\sum_{k=0}^{\infty} m_{n, k}\left(\frac{x}{b_{n}}\right) \sum_{j=0}^{k} m_{n, j}\left(\frac{x}{b_{n}}\right)-\sum_{k=0}^{\infty}\left(m_{n, k}\left(\frac{x}{b_{n}}\right)\right)^{2}\right| \\
& \leq\left|m_{n, k}\left(\frac{x}{b_{n}}\right) \sum_{k=0}^{\infty} m_{n, k}\left(\frac{x}{b_{n}}\right)\right| \leq \frac{1}{\sqrt{2 e}} \frac{1}{\sqrt{n \frac{x}{b_{n}}}} .
\end{aligned}
$$

Finally writing the estimates of (3.8) and (3.9) in (3.2), we get the result, given by (1.6). Thus the proof of the theorem is completed.

\section{Acknowledgments}

The author is thankful to the referees for their valuable remarks and suggestions leading to a better presentation of this paper.

Özet: Bu makalede, $\left[0, b_{n}\right]$ aralı̆̆ı üzerinde tanımlı fonksiyonlar için Chlodowsky tipi MKZD Operatörü olarak adlandırılan yeni bir tip MKZD Operatörü tanımlıyoruz ve olasılık teorisinin teknikleri vasıtasıyla, $\left[0, b_{n}\right](n \rightarrow \infty)$ iken sonsuza genişleyen aralık üzerinde 
sınırlı salınımlı olan fonksiyonlar için $M_{n}^{*}$ operatörünün yakınsaklık hızı üzerine bir tahmin veriyoruz.

\section{REFERENCES}

[1] Meyer-König, W., Zeller K., Bernsteinsche Potenzreihen, Studia Math.,19, (1960), 89-94.

[2] Guo, S., On the rate of convergence of the integrated Meyer-König and Zeller operators for functions of bounded variation, J.Approx. Theory, 56 (1989) 245-255.

[3] Guo, S., Degree of approximation to functions of bounded variation by certain operators, Approx. Theory and Its Appl., 4 (2) (1988) 9-18.

[4] Chen, W., On the integral type Meyer-König and Zeller type operators, Approx. Theory Appl., 2 (1986) 7-18.

[5] Zeng, Xiao-Ming, Rate of Convergence of bounded variation functions by two generalized Meyer-König and Zeller type operators, Comput. Math. Appl., 39 (2000) 1-13.

[6] Gupta, V., On a new type Meyer-König and Zeller type operators, J. Inequal. Pure and Appl. Math., 3 (4) (2002) 57.

[7] Gupta, V., On Bounded Variation Functions by General MKZD Operators, Acta Math. Sinica Eng. Seri., 23 (8) (2007), 1457-1462.

[8] Gupta, V., Karsli, H., Rate of convergence for the Bézier Variant of the MKZD Operators, Georgian Math. J. 14 (4) (2007), 651-659.

[9] Zeng, Xiao-Ming, Bounds for Bernstein basis functions and Meyer-König-Zeller basis functions, J. Math. Anal. Appl. 219 (1998) 364-376.

[10] Zeng, Xiao-Ming, Chen, W., On the rate of convergence of the generalized Durrmeyer type operators for functions of bounded variation, J. Approx. Theory, 102 (2000) 1-12.

[11] Bojanic, R., Vuilleumier, M., On the rate of convergence of Fourier Legendre series of functions of bounded variation, J. Approx. Theory 31 (1981) 67-79.

[12] Cheng, F., On the rate of convergence of Bernstein polynomials of functions of bounded variation, J. Approx. Theory 39 (1983) 259-274.

[13] Gupta, V., A sharp estimate on the degree of approximation to functions of bounded variation by certain operators, Approx. Theory and Its Appl., 11 (3) (1995) 106-107.

[14] Gupta V., Gupta, M. K., Rate of convergence for certain families of summation-integral type operators, J. Math. Anal. Appl. 296 (2004) 608-618.

[15] Karsli H. and Ibikli E., Rate of convergence of Chlodowsky-type Bernstein operators for functions of bounded variation, Numer. Funct. Anal. Optim., 28 (3-4) (2007), 367-378.

[16] Karsli H. and Ibikli E., Convergence rate of a new Bezier variant of Chlodowsky operators to bounded variation functions, J. Comput. Appl. Math., 212 (2) (2008), 431-443.

[17] Ibikli, E., Karsli, H., Rate of Convergence of Chlodowsky-type Durrmeyer Operators, J. Inequal. Pure and Appl. Math., Volume 6, Issue 4, (2005) Article 106.

Current address: Abant Izzet Baysal University, Faculty of Science and Arts, Department of Mathematics, 14280, Bolu/Turkey

E-mail address: fbharun@yahoo.com

$U R L:$ http://math.science.ankara.edu.tr 